\title{
Network-based analysis of differentially expressed genes in cerebrospinal fluid (CSF) and blood reveals new candidate genes for multiple sclerosis
}

\author{
Nahid Safari-Alighiarloo ${ }^{1}$, Mostafa Rezaei-Tavirani Corresp., ${ }^{1}$, Mohammad Taghizadeh ${ }^{2}$, Seyyed Mohammad \\ Tabatabaei $^{3}$, Saeed Namaki ${ }^{4}$ \\ ${ }^{1}$ Proteomics Research Center, Department of Basic Science, Faculty of Paramedical Sciences, Shahid Beheshti University of Medical Sciences, Tehran, \\ Iran \\ 2 Bioinformatics Department, Institute of Biochemistry and Biophysics, Tehran University, Tehran, Iran, Tehran, Iran \\ 3 Medical Informatics Department, Faculty of Paramedical Sciences, Shahid Beheshti University of Medical Sciences, Tehran, Iran \\ 4 Department of Immunology, Faculty of Medical Sciences, Shahid Beheshti University of Medical Sciences, Tehran, Iran \\ Corresponding Author: Mostafa Rezaei-Tavirani \\ Email address: tavirany@yahoo.com
}

Background: The involvement of multiple genes and missing heritability, which are dominant in complex diseases such as multiple sclerosis (MS), entail using network biology to more elucidate their molecular basis and genetic factors. We therefore aimed to integrate interactome (protein-protein interaction (PPI)) and transcriptomes data to construct and analyze PPI networks for MS disease. Methods: Gene expression profiles in paired cerebrospinal fluid (CSF) and peripheral blood mononuclear cells (PBMCs) samples from MS patients, sampled in relapse or remission and controls were analyzed.

Differentially expressed genes which determined only in CSF (MS vs. control) and PBMCs (relapse vs. remission) separately used to construct the Query-Query PPI (QQPPI) networks. The networks were further analysis to investigate more central genes, functional modules and complexes involved in MS progression. Results: The networks were analyzed and high centrality nodes were identified. Exploring of functional modules and complexes showed that the majority of high centrality nodes incorporated in biological pathways driving MS pathogenesis. Proteasome and spliceosome were also noticeable enriched pathways in PBMCs (relapse vs. remission) which identified by both modularity and clique analyses. Finally, STK4, RB1, CDKN1A, CDK1, RAC1, EZH2, SDCBP genes in CSF (MS vs. control) and CDC37, MAP3K3, MYC genes in PBMCs (relapse vs. remission) identified as potential candidate genes for MS, which were more central genes involved in biological pathways. Discussion: This study showed that network-based analysis could explicate the complex interplay between biological processes underlying MS. Furthermore an experimentally validation of candidate genes can lead to identification of potential therapeutic targets. 
Network-based analysis of differentially expressed genes in cerebrospinal fluid (CSF) and blood 1

reveals new candidate genes for multiple sclerosis

Nahid Safari-Alighiarloo ${ }^{1}$, Mostafa Rezaei-Tavirani ${ }^{1 *}$, Mohammad Taghizadeh ${ }^{2}$, Seyyed Mohammad Tabatabaei ${ }^{3}$, Saeed Namaki ${ }^{4}$

1- Proteomics Research Center, Department of Basic Science, Faculty of Paramedical Sciences, Shahid 7

Beheshti University of Medical Sciences, Tehran, Iran

2- Bioinformatics Department, Institute of Biochemistry and Biophysics, Tehran University, Tehran, Iran 9

3- Medical Informatics Department, Faculty of Paramedical Sciences, Shahid Beheshti University of 10

Medical Sciences, Tehran, Iran.

4- Department of Immunology, Faculty of Medical Sciences, Shahid Beheshti University of Medical

Correspond author*:

Mostafa Rezaei-Tavirani ${ }^{1}$

tavirany@yahoo.com telephone: +98 2122714248 
$\begin{array}{lr}\text { Abstract } & 30\end{array}$

Background: The involvement of multiple genes and missing heritability, which are dominant 31 in complex diseases such as multiple sclerosis (MS), entail using network biology to more 32

elucidate their molecular basis and genetic factors. We therefore aimed to integrate interactome 33 (protein-protein interaction (PPI)) and transcriptomes data to construct and analyze PPI networks 34 $\begin{array}{ll}\text { for MS disease. } & 35\end{array}$

Methods: Gene expression profiles in paired cerebrospinal fluid (CSF) and peripheral blood 36 mononuclear cells (PBMCs) samples from MS patients, sampled in relapse or remission and 37 controls were analyzed. Differentially expressed genes which determined only in CSF (MS vs. 38 control) and PBMCs (relapse vs. remission) separately used to construct the Query-Query PPI 39 (QQPPI) networks. The networks were further analysis to investigate more central genes, 40 functional modules and complexes involved in MS progression. 41

Results: The networks were analyzed and high centrality nodes were identified. Exploring of 42 functional modules and complexes showed that the majority of high centrality nodes 43 incorporated in biological pathways driving MS pathogenesis. Proteasome and spliceosome were 44 also noticeable enriched pathways in PBMCs (relapse vs. remission) which identified by both 45 modularity and clique analyses. Finally, STK4, RB1, CDKN1A, CDK1, RAC1, EZH2, SDCBP 46 genes in CSF (MS vs. control) and CDC37, MAP3K3, MYC genes in PBMCs (relapse vs. $\quad 47$ remission) identified as potential candidate genes for MS, which were more central genes 48 $\begin{array}{ll}\text { involved in biological pathways. } & 49\end{array}$

Discussion: This study showed that network-based analysis could explicate the complex 50 interplay between biological processes underlying MS. Furthermore an experimentally validation 51 of candidate genes can lead to identification of potential therapeutic targets. 52 
Introduction

Multiple sclerosis (MS) is a complex disease affecting the central nervous system (CNS) in

which genetic, environmental and immunological factors considered as its etiology (Ebers 2008;

Svejgaard 2008). Although MS shows both autoimmune and neurodegenerative features, the

pathophysiological processes which may occur both within and outside of the CNS remain

obscure and don't have an uniform distribution within the MS population (Brynedal et al. 2010).

To study such complex diseases, which involved noticeably missing heritability (Goris \& Liston

2012; Manolio et al. 2009), it is efficient to describe perturbed processes and dysregulated pathways rather to identify individual genes (Kim et al. 2011).

Transcriptome analysis of the target organ, i.e., the central nervous system, should reflect an

Since it is difficult to achieve CNS samples, cerebrospinal fluid (CSF) cells have been used in accessible and informative source of biological material in MS transcriptome studies (Achiron et al. 2004; Bomprezzi et al. 2003; Singh et al. 2007). In this line, it has been reported that the study 76 linking peripheral and CSF immune responses were essential to understand the immunopathogenesis of MS (Christensen et al. 2012).

Since the expression level change of a gene in a transcriptomic profile may be as a result of 79 an expression change of another gene and may not be the direct cause of the cellular phenotype, 80 additional information is required to put them in the context (Wachi et al. 2005). Network-based analyses of protein-protein interactions (PPI) or interactome delineate the known associations among proteins in context of biochemistry, signal transduction and biomolecular networks (Rezaei-Tavirani et al. 2016; Zali et al. 2014; Wu et al. 2009). The integration analysis of large scale gene expression and PPI data will place the differentially expressed genes in biological context (Bapat et al. 2010; Li et al. 2012; Safari-Alighiarloo et al. 2014; Safari-Alighiarloo et al. 86 2016). Protein networks reflect the functional grouping of interacting up/down regulated genes. 
The roles of the subsets of these genes, therefore, may be resolved using the combined data

(Wachi et al. 2005).

Recently, the topological analyses have been applied to PPI networks by tools or algorithms

such as modularity and centrality analyses by which the biological significance of proteins has

been determined (Huang et al. 2013; Lee et al. 2011). Graph centrality measures like degree,

betweenness and closeness centrality are so useful in the identification of nodes that are

functionally crucial in the network by ranking the elements of the network (Hindumathi et al.

2014). In the PPI network the nodes with high degree defined as hub proteins and the nodes with

high betweenness defined as bottleneck proteins, which both paly pivotal role in networks (Yu et

al. 2007). The interest gene sets which usually presented as gene modules, protein complexes or

This study integrated transcriptome-interactome data to construct PPI networks for MS

\section{Methods}

\section{Transcriptome data collection and processing}

Gene expression profiles in both CSF cells and PBMCs were obtained from the ArrayExpress

Database under the accession number of EMTAB- 69 based on the Human Genome 133 plus 2.0 
2004). After preprocessing, each expression profile containing 54, 675 probe sets that ones with

less discriminative power were removed according to the measurement of overall variance by the

Interactome data

The human PPI network was gathered from four major IMEx (Orchard et al. 2007) public

\section{QQPPI networks construction and topological analysis}

To construct Query-Query PPI (QQPPI) networks, the differentially expressed genes in CSF

$$
C_{B}(v)=\sum_{\mathrm{s} \neq \mathrm{t} \neq \mathrm{v} \in \mathrm{V}} \frac{\rho \mathrm{st}(\mathrm{v})}{\rho \mathrm{st}}
$$


Where the number of all shortest paths between node $s$ and $t$ regarded as $\rho s t$, and the number of

shortest paths which passing through a node $v$ out of $\rho s t$ regarded as $\rho s t(v)$. Indeed, this formula 146 represents the ratio of the number of shortest paths passing through node $v$ to the number of all shortest paths between $s$ and $t$. The current flow betweenness centrality of a node $\mathrm{v}$ is the average of the current flow over all source-target pairs. Closeness centrality is defined as the reciprocal 149 of the total distance from a node $\mathrm{v}$, to all other nodes. So, high values of closeness should 150 indicate that all other nodes are in proximity to node $\mathrm{v}$.

$$
C_{C}(v)=\frac{1}{\sum_{\mathrm{u} \in \mathrm{v}} \operatorname{dis}(\mathrm{u}, \mathrm{v})}
$$

The centroid value is the most complex node centrality index and is computed by focusing the calculus on couples of nodes $(\mathrm{v}, \mathrm{w})$. The centroid value of an individual node ' $\mathrm{v}$ ' is calculated by 154 considering the number of nodes that have minimum shortest path which are closer to ' $\mathrm{v}$ ' than ' $w$ '. A node $v$ with the highest centroid value is the node with the highest number of neighbors separated by the shortest path to $\mathrm{v}$,

$$
C_{c e n}(v)=\min (f(v, w))(5)
$$

Where $f(v, w)=y v(w)-y w(v)$ and $y v(w)$ denotes the number of node that are closer to $\mathrm{v}$ than importance.

$$
\lambda C_{I V}=A C_{I V}(6)
$$

Where $C_{I V}$ donates the Eigen vector and $\lambda$ donates the Eigen value.

Hub and bottleneck nodes were extracted from the networks in two steps; (1) In the networks,

Identification and annotation of functional modules

Network clustering was implemented by Clustering with overlap neighborhood expansion 
possible overlap (Nepusz et al. 2012). The modules were identified to have a minimum density

of $>0.05$ and a degree of $>5$. A cluster with a $p$-value of $<0.05$ was determined to be a module.

The functional meaning for identified modules was further explored, and they considered as

candidate functional modules if their genes were significantly enriched in biological process of

Identification of complexes containing clique

CFinder software was applied to extract biologically meaningful protein complexes from the PPI

Functional enrichment analysis

\section{Expression analysis}

We used Limma package to analyze gene expression profile, E-MTAB-69, for comparison of 
compared, 1163 differential expression genes with FDR $\leq 0.1$ were seen in PBMCs, but none in

Networks' topological analysis

We used only direct interactions of differentially expressed genes to construct QQPPI networks.

The ClusterONE algorithm was selectively implemented on CSF and PBMCs networks to mine 
signaling pathway, $\mathrm{T}$ cell receptor signaling pathway and Hematopoietic cell lineage. Further to

For PBMCs, enriched modules corresponded to the comparison of relapse versus remission

CFinder software was implemented to identify several three and four cliques in the QQPPI

For PBMCs (Relapse vs. remission), the identified complexes involved in many biological 
binding (ID: 351 and ID: 1181), protein targeting, sorting and translocation, protein transport and 258 homeostasis (ID:437), protein kinase (ID:5199), NIK-I-kappaB/NF-kappaB cascade and 259 cytokine activity (ID: 5269), apoptosis (ID: 5473, 5860, 5859, 5799 and 5800). 260

\section{Functional enrichment analysis of the networks}

To gain a full view of the networks potential functions, the networks' nodes were annotated

We screened more central nodes in CSF and PBMC to investigate detailed analysis about their 
functional modules and 13 (PSMA1, PSMA2, PSMA7, PSMB3, PSMD3, HNRNPM, FAS,

ACTB, CDC37, HSP90AB1, MAP3K3, IKBKE, CASP8) were associated in complexes.

Besides, it was obvious from the mining of functional modules and complexes that more central

The more central nodes and their expression values in CSF and PBMCs networks are illustrated

\section{Discussion}

Although myriad genetic studies investigating the MS pathogenesis, our understanding have genetic basis of complex disease by unraveling genes and processes not recognizable by genetic association approaches (Sharma et al. 2013; Yu et al. 2013). Due to implication of both intrathecal and peripheral immune activation in MS pathogenesis (Brynedal et al. 2010;

By studying two different tissues, neither being the actually affected tissue in MS, largely 
modules and complexes were characterized in these different tissues. More central genes

involved in immune response, apoptosis, cell cycle and cell adhesion pathways, which

The immunoproteasome is a cytokine-induced variant of the $20 \mathrm{~S}$ proteasome, which involved in

In this work, we also identified the candidate genes for MS employing of topological analysis 
pathway, cell cycle (M20), and it was downregulated. The evidence exists for the p21 as a cell-

cycle inhibitor that suppressed autoimmunity (Trakala et al. 2009). Indeed, recent studies

disclosed that p21 as a specific regulator of the homeostasis of memory/activated T lymphocytes

(Arias et al. 2007). The fourth marker, cyclin-dependent kinase 1 (CDK1) was enriched in p53 
cell-to-cell interactions such as SDCBP. They results indicated that $m i R-155 \_$- mice showed

lower levels of blood-brain barrier leakage in experimental autoimmune encephalomyelitis and an acute model of systemic inflammation (Lopez-Ramirez et al. 2014).

In case of PBMCs, the candidate markers corresponded to the comparison of relapse versus

remission state. The first candidate marker was cell division cycle 37 (CDC37). It was

CDC37 and HSP90 is a member of IKK complex that disruption of the interaction between

The last marker, v-myc avian myelocytomatosis viral oncogene homolog (MYC) incorporated in

\section{Conclusions}

This study showed the necessity of network-based analysis to get more insights in MS

pathogenesis at post-genomic era. In summary, QQPPI networks of abnormally expressed genes 
that genes with high centrality in the networks incorporated in main biological processes in MS

\section{References:}

Achiron A, Feldman A, Mandel M, and Gurevich M. 2007. Impaired Expression of Peripheral Blood Apoptotic-Related Gene Transcripts in Acute Multiple Sclerosis Relapse. Annals of the New York Academy of Sciences 1107:155-167 .

Achiron A, Gurevich M, Friedman N, Kaminski N, and Mandel M. 2004. Blood transcriptional signatures of multiple sclerosis: unique gene expression of disease activity. Annals of neurology 55:410417 .

Adamcsek B, Palla G, Farkas IJ, Derényi I, and Vicsek T. 2006. CFinder: locating cliques and overlapping modules in biological networks. Bioinformatics 22:1021-1023 .

Arias CF, Ballesteros-Tato A, García MI, Martín-Caballero J, Flores JM, Martínez-A C, and Balomenos D. 2007. p21CIP1/WAF1 controls proliferation of activated/memory T cells and affects homeostasis and memory T cell responses. The Journal of Immunology 178:2296-2306.

Bapat SA, Krishnan A, Ghanate AD, Kusumbe AP, and Kalra RS. 2010. Gene expression: protein interaction systems network modeling identifies transformation-associated molecules and pathways in ovarian cancer. Cancer research 70:4809-4819 .

Baranzini SE, Galwey NW, Wang J, Khankhanian P, Lindberg R, Pelletier D, Wu W, Uitdehaag BM, Kappos $\mathrm{L}$, and Polman $\mathrm{CH}$. 2009. Pathway and network-based analysis of genome-wide association studies in multiple sclerosis. Human molecular genetics 18:2078-2090 .

Basler M, Kirk CJ, and Groettrup M. 2013. The immunoproteasome in antigen processing and other immunological functions. Current opinion in immunology 25:74-80 .

Basler M, Mundt S, Bitzer A, Schmidt C, and Groettrup M. 2015. The immunoproteasome: a novel drug target for autoimmune diseases. Clin Exp Rheumatol 33:74-79 .

Basler M, Mundt S, Muchamuel T, Moll C, Jiang J, Groettrup M, and Kirk CJ. 2014. Inhibition of the immunoproteasome ameliorates experimental autoimmune encephalomyelitis. EMBO molecular medicine:e201303543.

Beekman JM, and Coffer PJ. 2008. The ins and outs of syntenin, a multifunctional intracellular adaptor protein. Journal of cell science 121:1349-1355.

Bomprezzi R, Ringnér M, Kim S, Bittner ML, Khan J, Chen Y, Elkahloun A, Yu A, Bielekova B, and Meltzer PS. 2003. Gene expression profile in multiple sclerosis patients and healthy controls: identifying pathways relevant to disease. Human molecular genetics 12 .2199-1912:

Brynedal B, Khademi M, Wallström E, Hillert J, Olsson T, and Duvefelt K. 2010. Gene expression profiling in multiple sclerosis: a disease of the central nervous system, but with relapses triggered in the periphery? Neurobiology of disease 37:6 .621-13 MINT, the molecular interaction database: 2009 update. Nucleic acids research:gkp983 . 
Chen G, and Goeddel DV. 2002. TNF-R1 signaling: a beautiful pathway. science 296:1634-1635 . Cellular sources of dysregulated cytokines in relapsing-remitting multiple sclerosis. Journal of neuroinflammation 9:1-12.

D’Ambrosi N, Rossi S, Gerbino V, and Cozzolino M. 2014. Rac1 at the crossroad of actin dynamics and neuroinflammation in Amyotrophic Lateral Sclerosis. Frontiers in cellular neuroscience 8 .

Dong C, Davis RJ, and Flavell RA. 2002. MAP kinases in the immune response. Annual review of immunology 20:55-72.

Ebers GC. 2008. Environmental factors and multiple sclerosis. The Lancet Neurology 7:268-277 .

Evsyukova I, Somarelli JA, Gregory SG, and Garcia-Blanco MA. 2010. Alternative splicing in multiple sclerosis and other autoimmune diseases. RNA biology 7:462-473 .

Gautier L, Cope L, Bolstad BM, and Irizarry RA. 2004. affy-analysis of Affymetrix GeneChip data at the probe level. Bioinformatics 20:307-315 .

Gentleman R, Carey V, Huber W, and Hahne F. 2011. Genefilter: Methods for filtering genes from microarray experiments. $R$ package version 1 .

Goris A, and Liston A. 2012. The immunogenetic architecture of autoimmune disease. Cold Spring Harbor perspectives in biology 4:a007260.

Hindumathi V, Kranthi T, Rao S, and Manimaran P. 2014. The prediction of candidate genes for cervix related cancer through gene ontology and graph theoretical approach. Molecular BioSystems 10:1450-1460.

Huang DW, Sherman BT, and Lempicki RA. 2008. Systematic and integrative analysis of large gene lists using DAVID bioinformatics resources. Nature protocols 4:44-57.

Huang K-C, Yang K-C, Lin H, Tsun-Hui TT, Lee W-K, Lee S-A, and Kao C-Y. 2013. Analysis of schizophrenia and hepatocellular carcinoma genetic network with corresponding modularity and pathways: novel insights to the immune system. BMC genomics 14:S10 .

Indovina P, Marcelli E, Casini N, Rizzo V, and Giordano A. 2013. Emerging roles of RB family: new defense mechanisms against tumor progression. Journal of cellular physiology 228:525-535 .

Junker BH, Koschützki D, and Schreiber F. 2006. Exploration of biological network centralities with CentiBiN. BMC bioinformatics 7:1.

Kebir H, Ifergan I, Alvarez JI, Bernard M, Poirier J, Arbour N, Duquette P, and Prat A. 2009. Preferential recruitment of interferon- $p$-expressing TH17 cells in multiple sclerosis. Annals of neurology 66:39.402-0

Kerrien S, Aranda B, Breuza L, Bridge A, Broackes-Carter F, Chen C, Duesbury M, Dumousseau M, Feuermann M, and Hinz U. 2011. The IntAct molecular interaction database in 2012. Nucleic acids research:gkr1088.

Kim Y-A, Wuchty S, and Przytycka TM. 2 .011ldentifying causal genes and dysregulated pathways in complex diseases. PLoS Comput Biol 7:e1001095 .

Lee S-A, Tsao TT, Yang K-C, Lin H, Kuo Y-L, Hsu C-H, Lee W-K, Huang K-C, and Kao C-Y. 2011. Construction and analysis of the protein-protein interaction networks for schizophrenia, bipolar disorder, and major depression. BMC bioinformatics 12:S20 .

Li J, and Jiang D. 2015. The role of epigenomics in the neurodegeneration of ataxia-telangiectasia. Epigenomics 7:137-141.

Li M, Wu X, Wang J, and Pan Y .2012 .Towards the identification of protein complexes and functional modules by integrating PPI network and gene expression data. BMC bioinformatics 13:109 .

Lopez-Ramirez MA, Wu D, Pryce G, Simpson JE, Reijerkerk A, King-Robson J, Kay O, de Vries HE, Hirst MC, and Sharrack B. 2014. MicroRNA-155 negatively affects blood-brain barrier function during neuroinflammation. The FASEB Journal 28:2551-2565 . 
Lynn DJ, Winsor GL, Chan C, Richard N, Laird MR, Barsky A, Gardy JL, Roche FM, Chan TH, and Shah N. 2008 .InnateDB: facilitating systems-level analyses of the mammalian innate immune response. Molecular systems biology 4 .

Manolio TA, Collins FS, Cox NJ, Goldstein DB, Hindorff LA, Hunter DJ, McCarthy MI, Ramos EM, Cardon LR, and Chakravarti A. 2009. Finding the missing heritability of complex diseases. Nature 461:747-753.

Maslov S, and Sneppen K. 2002. Specificity and stability in topology of protein networks. science 296:910-913.

Merico D, Isserlin R, Stueker O, Emili A, and Bader GD. 2010. Enrichment map :a network-based method for gene-set enrichment visualization and interpretation. PLoS One 5:e13984 .

Murga M, Fernández-Capetillo O, Field SJ, Moreno B, Luis R, Fujiwara Y, Balomenos D, Vicario A, Carrera AC, and Orkin SH. 2001. Mutation of E2F2 in mice causes enhanced T lymphocyte proliferation, leading to the development of autoimmunity. Immunity 15:959-970 .

Nepusz T, Yu H, and Paccanaro A. 2012. Detecting overlapping protein complexes in protein-protein interaction networks. Nature methods 9:471-472 .

Oeckinghaus A, and Ghosh S. 2009. The NF-kB family of transcription factors and its regulation. Cold Spring Harbor perspectives in biology 1:a000034 .

Orchard S, Kerrien S, Jones P, Ceol A, Chatr-aryamontri A, Salwinski L, Nerothin J, and Hermjakob H . .2007Submit your interaction data the IMEx way. Proteomics 7:28-34 .

Ray M, Ruan J, and Zhang W. 2008. Variations in the transcriptome of Alzheimer's disease reveal molecular networks involved in cardiovascular diseases. Genome Biol 9:R148 .

Rezaei-Tavirani M, Zamanian-Azodi M, Rajabi S, Masoudi-Nejad A, Rostami-Nejad M, and Rahmatirad S. 2016. Protein Clustering and Interactome Analysis in Parkinson and Alzheimer's Diseases. Archives of Iranian Medicine (AIM) 19.

Ruepp A, Waegele B, Lechner M, Brauner B, Dunger-Kaltenbach I, Fobo G, Frishman G, Montrone C, and Mewes H-W. 2010. CORUM: the comprehensive resource of mammalian protein complexes2009. Nucleic acids research 38:D497-D501.

Safari-Alighiarloo N, Taghizadeh M, and Rezaei-Tavirani M. 2015. CYTOSCAPE RETRIEVED PROTEINPROTEIN INTERACTION (PPI) NETWORKS: SEEKING THE CORRELATION OF HUMAN PROTEINS'TOPOLOGICAL FEATURES BETWEEN MAJOR PUBLIC PPI DATABASES DUE TO THEIR MEDICAL IMPORTANCE .International journal of analytical,pharmaceutical and biomedical sciences 4:137-146

Safari-Alighiarloo N, Taghizadeh M, Rezaei-Tavirani M, Goliaei B, and Peyvandi AA. 2014. Protein-protein interaction networks (PPI) and complex diseases. Gastroenterology and Hepatology from bed to bench 7: 9-16.

Safari-Alighiarloo N, Taghizadeh M, Tabatabaei SM, Shahsavari S, Namaki S, Khodakarim S, and Rezaei-Tavirani M. 2016. Identification of new key genes for type 1 diabetes through construction and analysis of the protein-protein interaction networks based on blood and pancreatic islet transcriptomes. Journal of Diabetes .

Salojin KV, Hamman BD, Chang WC, Jhaver KG, Al-Shami A, Crisostomo J, Wilkins C, Digeorge-Foushee AM, Allen J, and Patel N. 2014. Genetic deletion of Mst1 alters T cell function and protects against autoimmunity. PLoS One 9:e98151 .

Shannon P, Markiel A, Ozier O, Baliga NS, Wang JT, Ramage D, Amin N, Schwikowski B, and Ideker T. 2003. Cytoscape: a software environment for integrated models of biomolecular interaction networks. Genome research 13:2498-2504 .

Sharma A, Gulbahce N, Pevzner SJ ,Menche J, Ladenvall C, Folkersen L, Eriksson P, Orho-Melander M, and Barabási A-L. 2013. Network-based analysis of genome wide association data provides novel candidate genes for lipid and lipoprotein traits. Molecular \& Cellular Proteomics 12:3398-3408 . 
Singh M, Scott T, LaFramboise W, Hu F, Post J, and Ehrlich G. 2007. Gene expression changes in peripheral blood mononuclear cells from multiple sclerosis patients undergoing $\beta$-interferon therapy. Journal of the neurological sciences 258:52-59 .

Smyth GK .2005 .Limma: linear models for microarray data. Bioinformatics and computational biology solutions using $R$ and Bioconductor: Springer, 397-420.

Svejgaard A. 2008. The immunogenetics of multiple sclerosis. Immunogenetics 60:275-286 .

Zali H, Rezaei-Tavirani M. 201 .4Meningioma protein-protein interaction network. Archives of Iranian medicine 17:262 .

Trakala M, Arias CF, García MI, Moreno-Ortiz MC, Tsilingiri K, Fernández PJ, Mellado M, Díaz-Meco MT, Moscat J, and Serrano M. 2009. Regulation of macrophage activation and septic shock susceptibility via p21 (WAF1/CIP1). European journal of immunology 39:810-819.

Veroni C, Marnetto F, Granieri L, Bertolotto A, Ballerini C, Repice AM, Schirru L, Coghe G, Cocco E, and Anastasiadou E. 2015. Immune and Epstein-Barr virus gene expression in cerebrospinal fluid and peripheral blood mononuclear cells from patients with relapsing-remitting multiple sclerosis. Journal of neuroinflammation 12:1.

Wachi S, Yoneda K, and Wu R. 2005. Interactome-transcriptome analysis reveals the high centrality of genes differentially expressed in lung cancer tissues. Bioinformatics 21:4205-4208 .

Wu J, Vallenius T, Ovaska K, Westermarck J, Mäkelä TP, and Hautaniemi S. 2009. Integrated network analysis platform for protein-protein interactions .Nature methods 6:75-77.

Xenarios I, Salwinski L, Duan XJ, Higney P, Kim S-M, and Eisenberg D. 2002. DIP, the Database of Interacting Proteins: a research tool for studying cellular networks of protein interactions. Nucleic acids research 30:303-305.

Yoshida H, Kotani H, Kondo T, Tani I, Wei X, Tsuruta S, Kimura A, Asakawa M, Ito M, and Nagai S. 2013. CDK inhibitors suppress Th17 and promote iTreg differentiation, and ameliorate experimental autoimmune encephalomyelitis in mice. Biochemical and biophysical research communications 435:378-384 .

Yu F-D, Yang S-Y, Li Y-Y, and Hu W. 2013. Co-expression network with protein-protein interaction and transcription regulation in malaria parasite Plasmodium falciparum. Gene 518:7-16

555

557

Yu H, Kim PM, Sprecher E, Trifonov V, and Gerstein M. 2007. The importance of bottlenecks in protein networks: correlation with gene essentiality and expression dynamics. PLoS computational biology 3:e59 .

Zhang A. 2009. Protein interaction networks: computational analysis: Cambridge University Press. patients with multiple sclerosis. PLoS One 6:e16795 .

Zhu X, Gerstein M, and Snyder M. 2007. Getting connected: analysis and principles of biological networks. Genes \& development 21:1010-1024 . 
Figure 1

The degree distribution of nodes followed power law distribution.

(A) Degree distribution of differentially expressed genes in CSF QQPPI network. (B) PBMCs QQPPI network. The graph represents a decreasing trend of degree distribution with an increase in number of links showing scale-free topology.

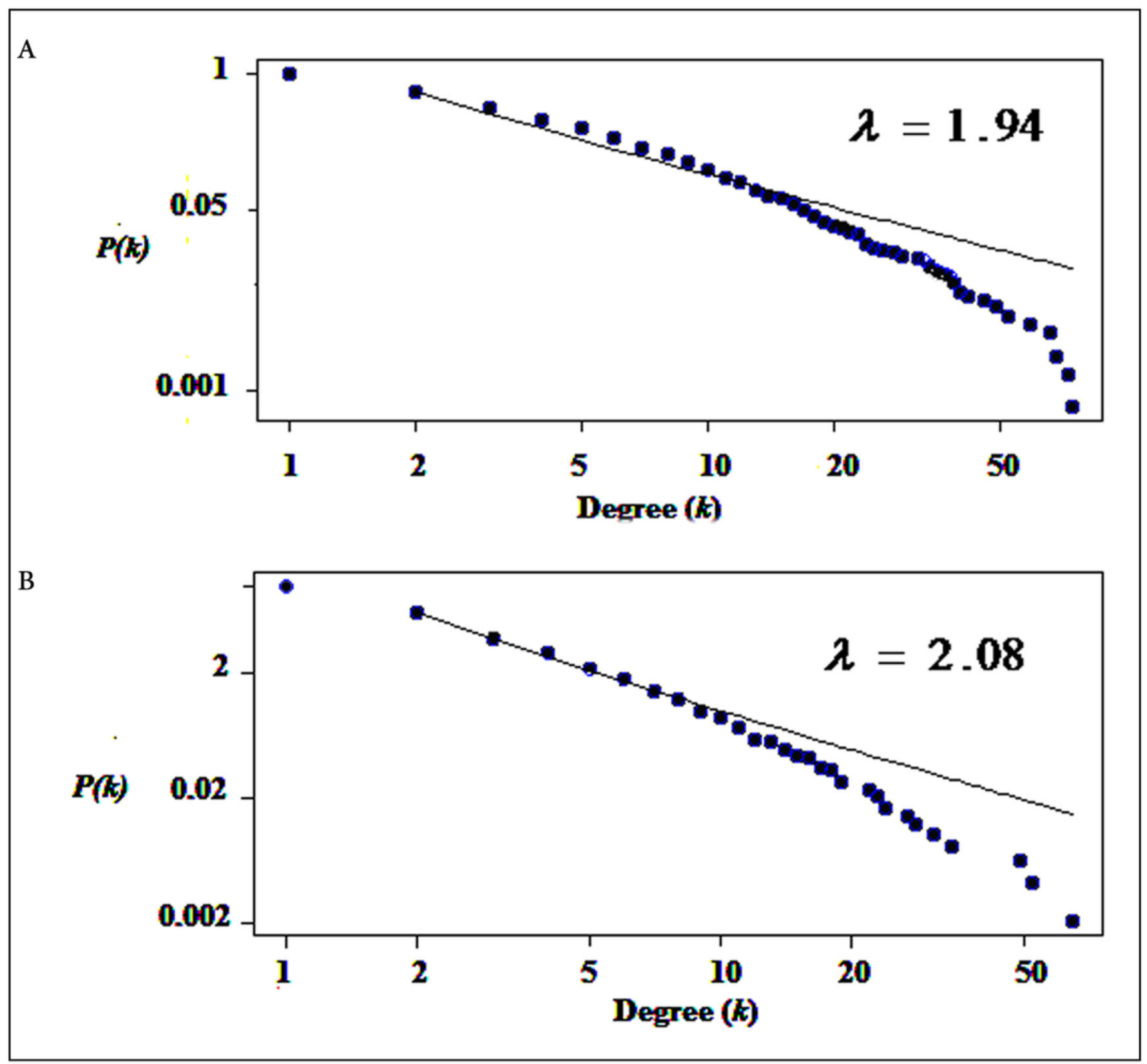


Figure 2

QQPPI networks generation by mapping of differentially expression genes on PPI data $\gg \mathrm{F} \diamond ?$

(A) CSF QQPPI network. (B) PBMCs QQPPI network. Nodes with high centrality measures are shown by bigger size than others. Green and red nodes represent proteins encoded by upand down-regulated genes, respectively. Graphical representation of nodes was implemented by "Spring Embedded" layout in Cystoscape. > $>\mathrm{mk}$ 


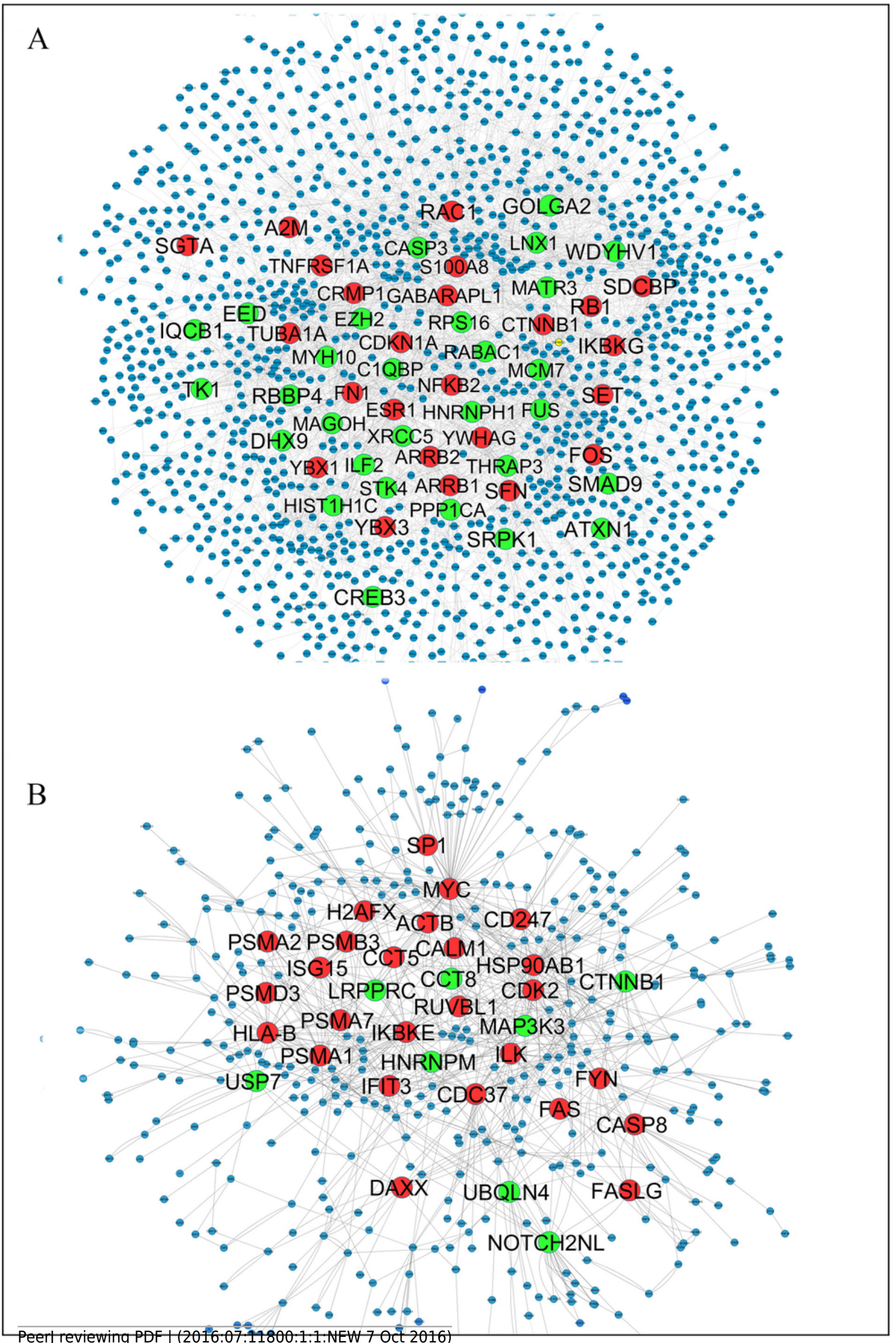




\section{Figure 3}

Functional categories of the networks were visualized using the Enrichment map plugin of the Cytoscape others $\mathrm{C}$

Significant biological processes are represented by one node in (A) CSF QQPPI network. (B) PBMCs QQPPI network. Nodes' sizes indicate the significance of the enrichment ( $P$-value).

Edges show gene overlap between nodes and thickness indicates the number of overlapping enriched genes. ente? $\gg \mathrm{Px}$

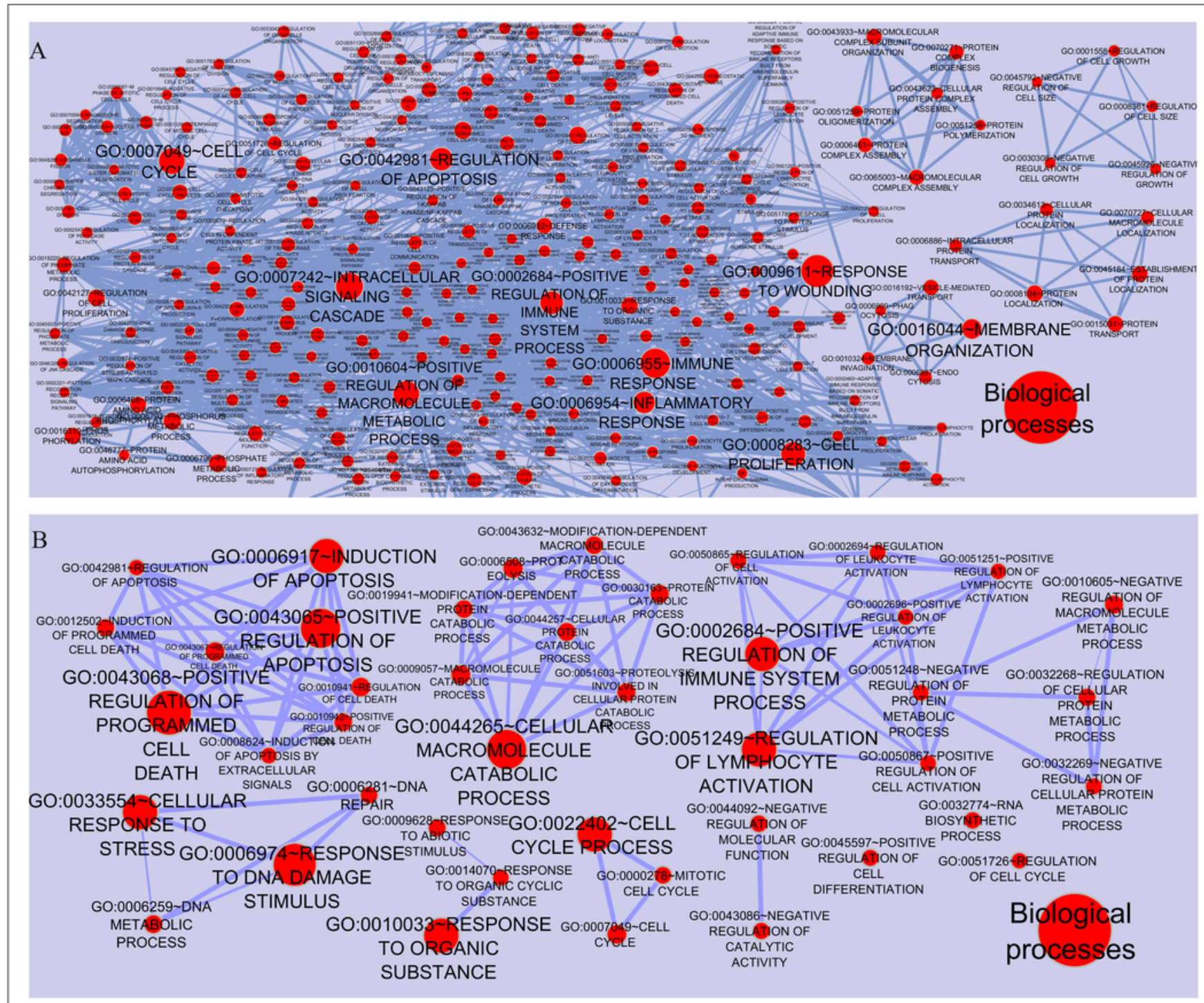




\section{Figure 4}

Nodes with high centrality measures and their expression values which involved in biological pathways $D>$

More central nodes in (A) CSF QQPPI network. (B) PBMCs QQPPI network. 


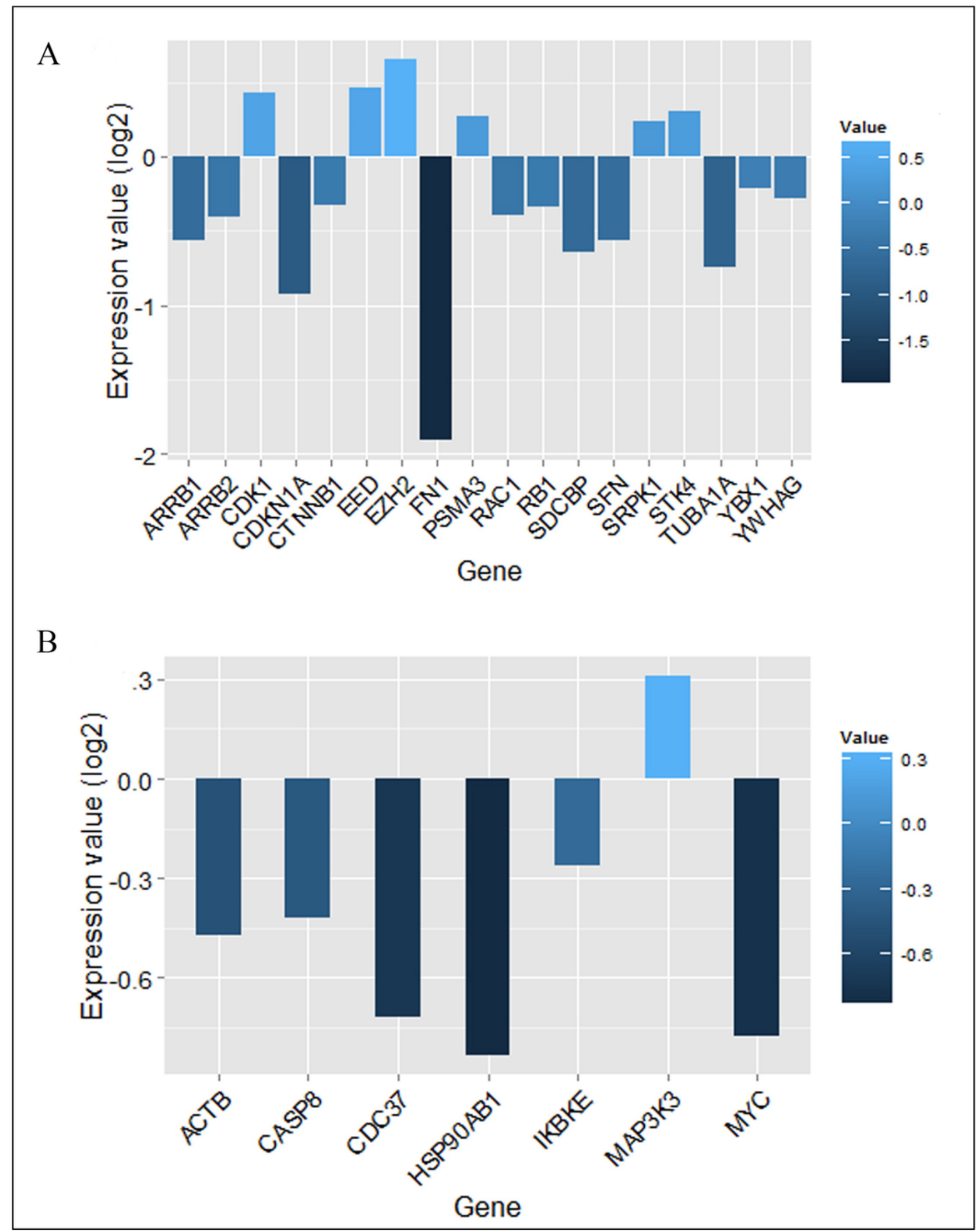


Figure 5

Candidate markers involved in functional modules and complexes. --EndFH

The functional enrichment of candidate markers in (A) CSF QQPPI network. (B) PBMCs QQPPI network. Modules and complexes illustrated by brown and blue dotted circles, respectively. ithem?
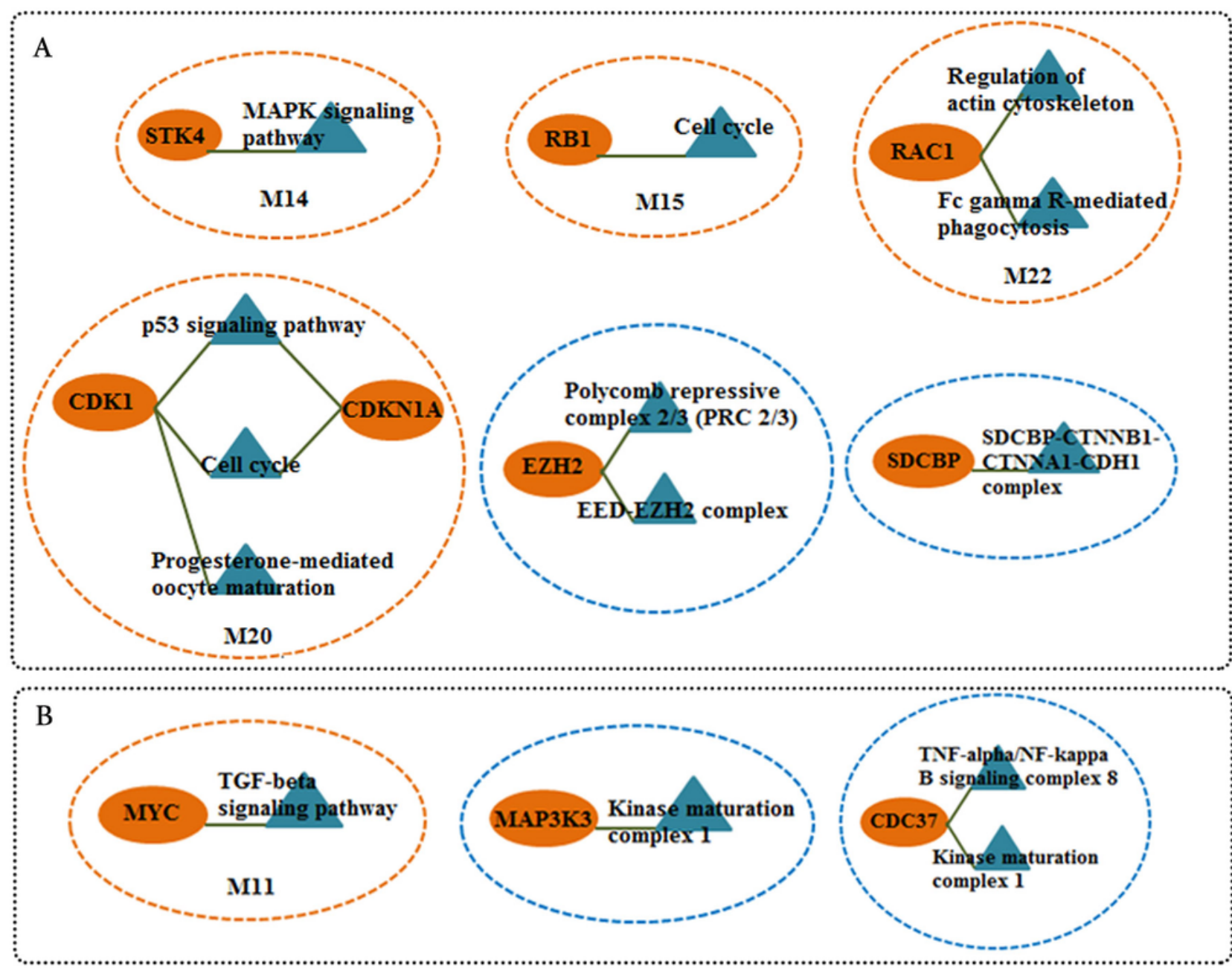


\section{Table 1 (on next page)}

Hub-bottleneck identification.

Cut-off determination for hubs \& number of hubs and bottlenecks. 
Table 1 Hub-bottleneck identification. Cut-off determination for hubs \& number of hubs and bottlenecks.

\begin{tabular}{lllccc}
\hline & Mean (M) & $\begin{array}{l}\text { Standard } \\
\text { Deviation (S.D) }\end{array}$ & Cut-off (M+2*S.D) & $\begin{array}{l}\text { Number } \\
\text { of hubs }\end{array}$ & $\begin{array}{l}\text { Number of } \\
\text { bottlenecks }\end{array}$ \\
\hline CSF & 4.86 & 2.73 & 19.4 & 56 & 72 \\
PBMCs & 3.89 & 6.06 & 16.01 & 20 & 25 \\
\hline
\end{tabular}




\section{Table 2 (on next page)}

Modularity analysis.

The list of pathways enriched in modules for CSF (MS vs. controls). 
Table 2 Modularity analysis. The list of pathways enriched in modules for CSF (MS vs. controls).

\begin{tabular}{lll}
\hline Module ID & \multicolumn{1}{c}{ Pathway } & p-value \\
\hline M1 & hsa04062: Chemokine signaling pathway & $7.7 \mathrm{E}-3$ \\
& hsa04060: Cytokine-cytokine receptor interaction & $1.5 \mathrm{E}-2$ \\
M2 & hsa04672: Intestinal immune network for IgA production & $3.8 \mathrm{E}-2$ \\
& hsa05010: Alzheimer's disease & $3.0 \mathrm{E}-3$ \\
& hsa05014: Amyotrophic lateral sclerosis (ALS) & $3.1 \mathrm{E}-2$ \\
M4 & hsa04720: Long-term potentiation & $4.0 \mathrm{E}-2$ \\
& hsa04640: Hematopoietic cell lineage & $4.7 \mathrm{E}-6$ \\
& hsa04060: Cytokine-cytokine receptor interaction & $1.4 \mathrm{E}-4$ \\
& hsa04210: Apoptosis & $8.6 \mathrm{E}-4$ \\
& hsa05020: Prion diseases & $2.1 \mathrm{E}-2$ \\
h5 & hsa05332: Graft-versus-host disease & $2.3 \mathrm{E}-2$ \\
M9 & hsa04940: Type I diabetes mellitus & $2.5 \mathrm{E}-2$ \\
M13 & hsa04620: Toll-like receptor signaling pathway & $3.3 \mathrm{E}-2$ \\
& hsa05217: Basal cell carcinoma & $1.7 \mathrm{E}-5$ \\
M14 & hsa04340: Hedgehog signaling pathway & $2.3 \mathrm{E}-3$ \\
M15 & hsa04010: MAPK signaling pathway & $2.3 \mathrm{E}-3$ \\
M20 & hsa04110: Cell cycle & $1.5 \mathrm{E}-2$ \\
& hsa04115: p53 signaling pathway & $1.2 \mathrm{E}-2$ \\
M22 & hsa04110: Cell cycle & $3.3 \mathrm{E}-10$ \\
M23 & hsa04914: Progesterone-mediated oocyte maturation & $2.5 \mathrm{E}-8$ \\
& hsa04810: Regulation of actin cytoskeleton & $1.8 \mathrm{E}-3$ \\
& hsa04666: Fc gamma R-mediated phagocytosis & $1.6 \mathrm{E}-2$ \\
& hsa04530: Tight junction & $2.4 \mathrm{E}-2$ \\
& hsa04144: Endocytosis & $4.4 \mathrm{E}-2$ \\
& hsa04540: Gap junction & $1.5 \mathrm{E}-2$ \\
& hsa04510: Focal adhesion & $2.7 \mathrm{E}-2$ \\
& & $2.7 \mathrm{E}-2$ \\
& & $3.1 \mathrm{E}-2$ \\
\hline & & \\
& &
\end{tabular}




\section{Table 3 (on next page)}

Modularity analysis.

The list of pathways enriched in modules for PBMCs (relapse vs. remission) 
Table 3 Modularity analysis. The list of pathways enriched in modules for PBMCs (relapse vs. remission)

\begin{tabular}{lll}
\hline Module ID & \multicolumn{1}{c}{ Pathway } & $p$-value \\
\hline M1 & hsa04612: Antigen processing and presentation & $6.6 \mathrm{E}-8$ \\
& hsa05340: Primary immunodeficiency & $2.7 \mathrm{E}-2$ \\
& hsa05332: Graft-versus-host disease & $3.0 \mathrm{E}-2$ \\
& hsa02010: ABC transporters & $3.4 \mathrm{E}-2$ \\
M7 & hsa04115: p53 signaling pathway & $3.5 \mathrm{E}-3$ \\
& hsa04110 : Cell cycle & $1.2 \mathrm{E}-2$ \\
M8 & hsa04623: Cytosolic DNA-sensing pathway & $2.5 \mathrm{E}-4$ \\
& hsa04622: RIG-I-like receptor signaling pathway & $5.2 \mathrm{E}-4$ \\
& hsa04620: Toll-like receptor signaling pathway & $1.5 \mathrm{E}-3$ \\
M9 & hsa04120: Ubiquitin mediated proteolysis & $2.3 \mathrm{E}-2$ \\
M10 & hsa03050 : Proteasome & $1.3 \mathrm{E}-9$ \\
M11 & hsa03040: Spliceosome & $1.0 \mathrm{E}-3$ \\
& hsa04350 :TGF-beta signaling pathway & $4.1 \mathrm{E}-2$ \\
\hline
\end{tabular}




\section{Table 4(on next page)}

Clique analysis fai

The list of complexes enriched for CSF and PBMCs n 
Table 4 Clique analysis. The list of complexes enriched for CSF and PBMCs

\begin{tabular}{|c|c|}
\hline Module ID & Complex \\
\hline \multicolumn{2}{|l|}{ CSF (MS vs. controls) } \\
\hline $\begin{array}{l}\text { PSMA3, PSMB1, PSMB3, PSMB9, PSME1, } \\
\text { PSMD7 }\end{array}$ & Proteasome (ID:39, 192,193) \\
\hline GPS2, NCOR2, TBL1X & SMRT complex (ID:58) \\
\hline EED, EZH2, RBBP4 & $\begin{array}{l}\text { Polycomb repressive complex } 2,3 \text { (PRC 2,3) } \\
\text { (ID: } 105,996,995), \text { EED-EZH2 complex } \\
\text { (ID:974), }\end{array}$ \\
\hline CCNB1, CCNB2, CCND1, CDK1, CDKN1A & Cell cycle kinase complex CDC2 (ID:310) \\
\hline CCND1,CCND3, CDKN1A & Cell cycle kinase complex CDK5 (ID:313) \\
\hline CBX5, DSN1, ZWINT & Mis 12 centromere complex (ID:1464) \\
\hline CTNNA1, CTNNB1, SDCBP & $\begin{array}{l}\text { SDCBP-CTNNB1-CTNNA1-CDH1 complex } \\
\text { (ID:1839) }\end{array}$ \\
\hline TUBA1A, TUBA1B, TUBA1C & 60S APC containing complex (ID:3008) \\
\hline $\begin{array}{l}\text { IGF2BP1, ILF2, NOLC1, RPLP2, RPS11, RPS16, } \\
\text { SRPK1, TUBA1A, YBX1 }\end{array}$ & $\begin{array}{l}\text { Nop56p-associated pre-rRNA complex } \\
\text { (ID:3055) }\end{array}$ \\
\hline MAP2K1, SFN, YWHAG & $\begin{array}{l}\text { Ksr1 complex (Ksr1, Mek, 14-3-3, Mapk), } \\
\text { EGF stimulated (ID:5909, 5886) }\end{array}$ \\
\hline \multicolumn{2}{|l|}{ PBMCs (Relapse vs. Remission) } \\
\hline $\begin{array}{l}\text { PSMA1, PSMA2, PSMA7, PSMB10, PSMB3, } \\
\text { PSMD3, PSMD4 }\end{array}$ & $\begin{array}{l}\text { Proteasome (ID: } 38,39,181,191,192,193 \text {, } \\
\text { 194) }\end{array}$ \\
\hline DHX15, PABPC1, PRPF19, SF3B3, SNRPB & Spliceosome (ID:351) \\
\hline ACTB, ANXA6, MYH9, SPTAN1 & PA700-20S-PA28 complex (ID:437) \\
\hline $\begin{array}{l}\text { HNRNPH1, HNRNPM, PABPC1, PRPF19, } \\
\text { SF3B3, SNRPB }\end{array}$ & C complex spliceosome (ID:1181) \\
\hline CDC37, HSP90AB1, MAP3K3 & Kinase maturation complex 1 (ID:5199) \\
\hline CDC37, HSP90AB1, IKBKE & $\begin{array}{l}\text { TNF-alpha/NF-kappa B signaling complex } 8 \\
\text { (ID: 5269) }\end{array}$ \\
\hline CASP8 FADD FAS & $\begin{array}{l}\text { FAS-FADD-CASP8 complex (ID: } 5473 \text {, } \\
\text { 5860), FAS-FADD-CASP8-CASP10 complex } \\
\text { (ID: 5859), Death induced signaling complex } \\
\text { DISC (ID: } 5799,5800 \text { ) }\end{array}$ \\
\hline ACTB, MYH9, SPTAN1 & Emerin complex 1 (ID: 5604) \\
\hline
\end{tabular}




\section{Table 5 (on next page)}

Central genes ost? ?

The list of more central genes enriched in functional modules and complexes for CSF and PBMCs $\curvearrowright=\curvearrowright$ ? $\mathrm{Xj}$ 
Table 5 Central genes. The list of more central genes enriched in functional modules and complexes for CSF and PBMCs

\begin{tabular}{ll}
\hline Module/complex ID & Gene symbol \\
\hline CSF(MS $\boldsymbol{v s}$. controls) & \\
M13 & SMAD1 \\
M14 & STK4 \\
M15 & RB1 \\
M20 & CDKN1A, CDK1 \\
M22 & RAC1 \\
M23 & ARRB2, ARRB1 \\
M25 & FN1 \\
ID:39, 192,193 & PSMA3 \\
ID:105, $974,996,995$ & EED, EZH2, RBBP4 \\
ID:310,313 & CDK1, CDKN1A \\
ID: 1839 & CTNNB1, SDCBP \\
ID:3008 & TUBA1A \\
ID:3055 & SRPK1, YBX1, ILF2, RPS16 \\
ID:5909, 5886 & SFN, YWHAG \\
PBMCs (relapse vs. remission & \\
M7 & CDK2 \\
M8 & IKBKE \\
M10 & PSMA1 \\
M11 & MYC \\
ID: $38,39,181,191,192,193,194$ & PSMA1, PSMA2, PSMA7, PSMB3, PSMD3 \\
ID:1181 & HNRNPM \\
ID:437,5604 & ACTB \\
ID:5199, 5269 & CDC37, HSP90AB1, MAP3K3, IKBKE \\
ID: $5473,5860,5859,5799,5800$ & CASP8,FAS \\
\hline
\end{tabular}

